\title{
The Crystal Structure of $\mathrm{CsMnCl}_{3}, 2 \mathrm{H}_{2} \mathrm{O}$
}

\author{
STIG JORGO JENSEN and PALLE ANDERSEN \\ Department of Technology, The Royal Dental College, Aarhus, Denmark, and
}

SVEND ERIK RASMUSSEN

Department of Inorganic Chemistry, University of Aarhus, Aarhus, Denmark

\begin{abstract}
$\mathrm{CsMnCl}_{3}, 2 \mathrm{H}_{2} \mathrm{O}$ is orthorhombic; the space-group is $P c c a-D_{2 \mathrm{~h}}^{8}$ with $a=9.060 \AA, b=7.285 \AA, c=11.455 \AA$ and four formula units per unit cell. The structure was determined by two-dimensional Patterson projections and was refined by Fourier difference synthesis. Each manganese atom is octahedrally coordinated to four chlorine atoms and two water molecules. The water molecules occupy cis-positions.
\end{abstract}

In connection with investigations of the structures of the double-salts of $\mathrm{I}_{\mathrm{CsCl}}$ and anhydrous $\mathrm{MnCl}_{2}$ of Andersen ${ }^{1}$ we investigated some hydrates as well. We prepared $\mathrm{CsMnCl}_{3}, 2 \mathrm{H}_{2} \mathrm{O}$ and $\mathrm{Cs}_{2} \mathrm{MnCl}_{4}, 2 \mathrm{H}_{2} \mathrm{O}$. The existence of the latter was reported by Vermande ${ }^{2}$. Of particular interest was the coordination around the manganous ion and the location of the water molecules. This paper presents the result of the crystal structure analysis of $\mathrm{CsMnCl}_{3}, 2 \mathrm{H}_{2} \mathrm{O}$. The structure analysis of the other hydrate is in progress.

\section{EXPERIMENTAL}

$\mathrm{CsMnCl}_{3}, 2 \mathrm{H}_{2} \mathrm{O}$ was prepared from an aqueous solution containing 0.01 mole $\mathrm{CsCl}$ and 0.01 mole $\mathrm{MnCl}_{2}, 4 \mathrm{H}_{2} \mathrm{O}$ (Merck p.a.) and slow evaporation at $20^{\circ} \mathrm{C}$. The crystals were rectangular or eight-sided pink prisms. Chemical analysis of the compound gave the following results: $\mathrm{Mn} 16.59 ; \mathrm{Cl} 32.28 ; \mathrm{H}_{2} \mathrm{O} 10.92 ; \mathrm{Cs} 40.21$. Calc. for $\mathrm{CsMnCl}_{3}, 2 \mathrm{H}_{2} \mathrm{O}$ : $\mathrm{Mn}$ 16.63; $\mathrm{Cl} 32.22 ; \mathrm{H}_{2} \mathrm{O} 10.90 ; \mathrm{Cs} 40.25$. The Mn-content was determined by complexometric titration with EDTA, Cl by potentiometric titration using $\mathrm{AgNO}_{3}$, and the water gravimetrically by heating the compound to $100^{\circ} \mathrm{C}$. The crystals decompose at $58^{\circ} \mathrm{C}$. Cs was calculated as the balance. The density was $2.84 \mathrm{~g} / \mathrm{cm}^{3}\left(20^{\circ} \mathrm{C}\right)$ as measured by flotation in a mixture of carbon tetrachloride and acetylene tetrabromide.

The unit-cell dimensions were determined from a Guinier powder pattern using cesiumchloride as a reference. FeKa radiation was employed. The lattice constant was found to be $4.122 \AA$ ( $\mathrm{Fe} K \alpha=1.93597 \AA)$ from Bradley-Jay powder diagrams using a $19 \mathrm{~cm}$ camera. The Nelson, Riley ${ }^{3}$ extrapolation procedure was used for this determination of $a_{\mathrm{CsCl}}$.

Acta Chem. Scand. 16 (1962) No. 8 
For intensity measurements, a crystal of the dimensions $100 \mu \times 40 \mu \times 20 \mu$ was mounted on a Weissenberg goniometer and oriented along the $a$-axis. The $h 0 l$ and $h k 0$ reflexions were recorded with a Precession goniometer, the $0 k l$ reflexions with an integrating Retigraph goniometer. Zr-filtered molybdenum radiation was used in all cases. The Precession-films were evaluated visually by means of a standard intensity-scale and the Retigraph-films with a densitometer. Corrections for Lorentz- and polarization factors were made using charts. No correction for absorption was made. The linear absorption coefficient is $76 \mathrm{~cm}^{-2}$.

\section{STRUCTURE DETERMINATION}

The conditions limiting reflexions are the following:

$h k l$ : no conditions; $0 k l: l=2 n ; h 0 l: l=2 n ; h k 0: h=2 n$. Consequently the space group is No. 54, Pcca- $D_{2 \mathrm{~h}}^{8}$.

The unit cell contains four units of $\mathrm{CsMnCl}_{3}, 2 \mathrm{H}_{2} \mathrm{O}$. The general position in Pcca is eight-fold. Thus the Cs-atom, the Mn-atom and one of the Cl-atoms must occupy some of the special four-fold positions of the space group.

The parameters of the cesium and of the manganese atoms were obtained from 100 and 010 Patterson projections. The cesium atom was placed in the four-fold position $1 / 4,0, z$ using the value 0.146 for $z$. The manganous ion was put into the other four-fold position $0, y, 1 / 4$ using for $y 1 / 2$. The positions of the symmetry-related manganous ions are thus: $0,1 / 2,1 / 4 ; 0,1 / 2,3 / 4 ; 1 / 2,1 / 2,3 / 4$; $1 / 2,1 / 2,1 / 4$. The positions of the chlorine ions were ambigous from the Patterson projections although their correct positions are clearly indicated on the Patterson maps.

Information on the positions of the lighter atoms was obtained from Fourier projections along the $a$ - and $b$-axes. The signs of the structure factors $F(0 k l)$ and $F(h 0 l)$ were calculated from the contributions of the Cs-atoms and the Mn-atoms. The positions of the chlorine atoms were clearly indicated on the Fourier maps. The positions of the oxygen atoms were, however, obscured by the chlorine atoms.

Difference Fourier projections were made incorporating the positions of the $\mathrm{Cs}, \mathrm{Mn}$ and $\mathrm{Cl}$ atoms in the structure factor calculations. The difference projections were of the variety found rarely outside textbooks: The oxygen atoms appeared clearly, one set of chlorine atoms was seen on a gradient. The Mn-atoms which were assumed preliminary to overlap completely in the $0 \mathrm{kl}$ projection at $y=1 / 2$ must obviously be moved to the positions $y=1 / 2+\delta$ and $y=1 / 2-\delta$.

Fig. 1 shows the first $0 k l$ difference projection and Fig. 2 the first $h 0 l$ difference projection. The refinement was stopped after 3 cycles of difference Fourier projections on each of the $0 k l$ and the $h 0 l$ projections.

The $h k 0$ structure factors were not used for refinement. The agreement between observed and calculated structure factors is as good for this projections as for the other ones (Table 1).

Part of the computations were carried out by aid of a Fourier analogue computer of the Hägg-Laurent type built by Frank ${ }^{4}$. Most of the structure factor and Fourier-computations were carried out on the electronic digital computer DASK of Regnecentralen, Copenhagen.

Acta Chem. Scand. 16 (1962) No. 8 


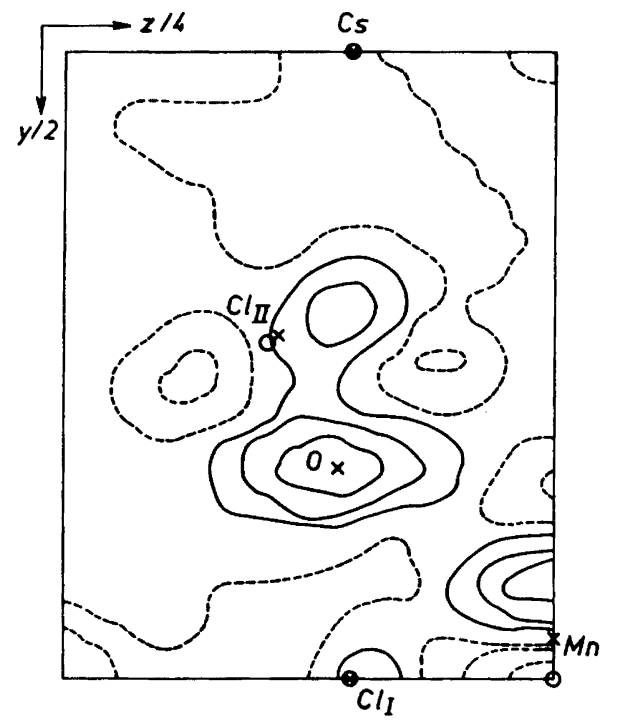

Fig. 1. First difference synthesis. Projection on (100). The contours are at arbitrary intervals. The broken lines represent zero and negative contours. The small circles show the atom locations derived from the Fourier projections and the small crosses show the final locations. The map shows the place of the oxygen atom, the errors in the locations of $\mathrm{Cl}_{\mathrm{II}}$ and $\mathrm{Mn}$ and the too high temperature factor of the $\mathrm{Cl}_{\mathrm{I}}$-atom.

The following expression was used for calculating the atomic scattering factors:

$$
\begin{gathered}
f_{n}(\sin \Theta / \lambda)=A_{n} \exp \left[-a_{n}(\sin \Theta / \lambda)^{2}\right]+B_{n} \exp \left[-b_{n}(\sin \Theta / \lambda)^{2}\right] \\
+C_{n} \exp \left[-c_{n}(\sin \Theta / \lambda)^{2}\right]
\end{gathered}
$$

The following parameters which include isotropic temperature factors were used:

\begin{tabular}{lrrrcccc} 
& $A$ & \multicolumn{1}{c}{$B$} & \multicolumn{1}{c}{$C$} & $a$ & $b$ & $c$ & Ref. \\
$\mathrm{Cs}$ & 18.965 & 20.752 & 14.659 & 3.358 & 5.444 & 21.179 & 5 \\
$\mathrm{Mn}$ & 8.630 & 9.427 & 6.744 & 2.145 & 5.524 & 28.341 & 5 \\
$\mathrm{Cl}_{\mathrm{I}}$ & 7.511 & 7.487 & 1.881 & 4.128 & 34.56 & 2.80 & 6 \\
$\mathrm{Cl}_{\mathrm{II}}$ & 7.511 & 7.487 & 1.881 & 4.428 & 34.86 & 3.10 & 6 \\
$\mathrm{O}$ & 2.113 & 4.637 & 1.211 & 5.967 & 17.85 & 3.10 & 6
\end{tabular}

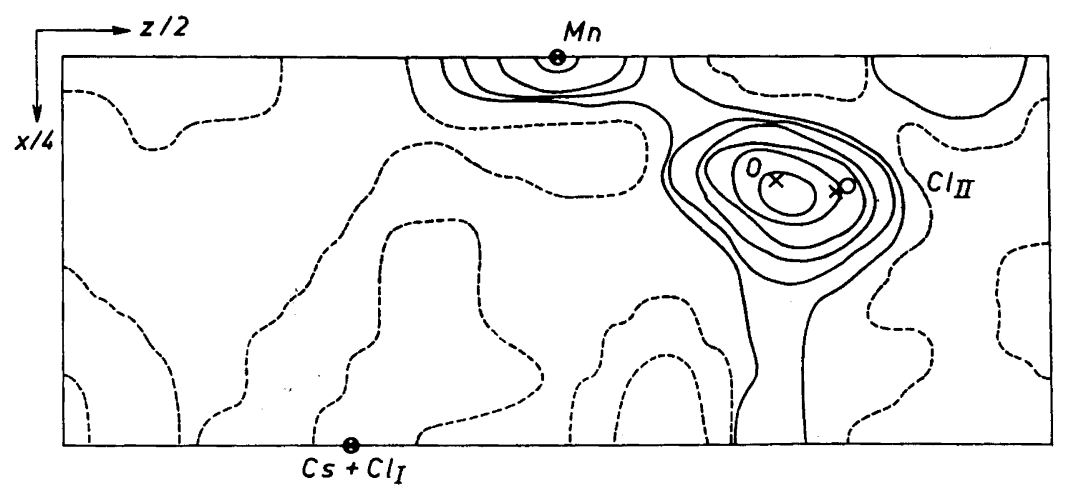

Fig. 2. First difference synthesis. Projection on (010). The map shows the place of the oxygen atom, the error in the location of $\mathrm{Cl}_{\mathrm{II}}$ and the too high temperature factor of the Mn-atom. 
The programmes were written according to descriptions of Westman, Blomqvist and Åsbrink ${ }^{5}$ by Per Mondrup and Finn Larsen of Regnecentralen.

$$
\text { Crystal data }
$$

For convenience all pertinent crystal data are presented below:

Crystal system: orthorhombic

$$
\begin{aligned}
& a=9.060 \pm 0.005 \AA \\
& b=7.285 \pm 0.005 \AA \\
& c=11.455 \pm 0.005 \AA
\end{aligned}
$$

Space group: No. 54, Pcca $-D_{2 \mathrm{~h}}^{8}$

Density, calculated (four formula units in the elementary cell): $2.89 \mathrm{~g} / \mathrm{cm}^{3}$. Density, measured: $2.84 \mathrm{~g} / \mathrm{cm}^{3}$

Absorption coefficient for Mo $K \alpha$ radiation: $76 \mathrm{~cm}^{-1}$

$F(000)=583$

Residual factor $R=\Sigma\left\|F_{\mathrm{o}}|-| F_{\mathrm{c}}\right\| / \Sigma\left|F_{\mathrm{o}}\right| ; R_{h 0 l}=10.1 \% ; R_{h k 0}=9.5 \%$; $R_{0 k l}=9.3 \%$, including all reflexions with $\sin \Theta<0.5$.

Non-observed reflexions are included with half the value of the smallest observed $F(h k l)$ value.

Atomic parameters and temperature factors:

$\begin{array}{lcccc}\text { Atom } & x & y & z & B \\ \mathrm{Cs} & 0.250 & 0.000 & 0.146 & 3.15 \\ \mathrm{Mn} & 0.000 & 0.467 & 0.250 & \mathbf{1 . 8 0} \\ \mathrm{Cl}_{\mathrm{I}} & 0.250 & 0.500 & 0.146 & 2.80 \\ \mathrm{Cl}_{\text {II }} & 0.085 & 0.228 & 0.391 & \mathbf{3 . 1 0} \\ \mathrm{O} & 0.080 & 0.669 & 0.361 & \mathbf{3 . 1 0}\end{array}$

Interatomic distances (in $\AA$ ):

Within octahedrons:

$\mathrm{Mn}-\mathrm{Cl}_{\mathrm{I}} \quad 2.57$

$\mathrm{Mn}-\mathrm{Cl}_{\mathrm{II}} \quad 2.50$

$\mathrm{Mn}-\mathrm{O} \quad 2.08$

$\mathrm{Cl}_{\mathrm{I}}-\mathrm{Cl}_{\mathrm{II}} \quad \mathbf{3 . 7 5}$

$\mathrm{Cl}_{\mathrm{I}}-\mathrm{Cl}_{\mathrm{II}} \quad \mathbf{3 . 6 4}$

$\mathrm{Cl}_{\mathrm{II}}-\mathrm{Cl}_{\mathrm{II}} \quad 3.58$

$\mathrm{Cl}_{\mathrm{II}}-\mathrm{O} \quad 3.23$

$\mathrm{Cl}_{\mathrm{I}}-\mathrm{O} \quad 3.15$

$\mathrm{Cl}_{\mathrm{I}}-\mathrm{O} \quad 3.24$

$\mathrm{O}^{\mathrm{I}}-\mathrm{O} \quad 2.92$

From the cesium - atom:

$\begin{array}{ll}\mathrm{Cs}-\mathrm{Cl}_{\mathrm{I}} & 3.64 \\ \mathrm{Cs}-\mathrm{Cl}_{\text {II }} & 3.59 \\ \mathrm{Cs}-\mathrm{Cl}_{\mathrm{II}} & \mathbf{3 . 6 7} \\ \mathrm{Cs}-\mathrm{Cl}_{\text {II }} & \mathbf{3 . 4 8}\end{array}$

Between neighbouring octahedrons:

$\begin{array}{ll}\mathrm{Cl}_{\mathrm{I}}-\mathrm{Cl}_{\mathrm{II}} & 3.83 \\ \mathrm{Cl}_{\mathrm{II}}-\mathrm{Cl}_{\mathrm{II}} & 4.46 \\ \mathrm{Cl}_{\mathrm{II}}-\mathrm{Cl} & 4.43 \\ \mathrm{Cl}_{\mathrm{II}}-\mathrm{O} & 3.14 \\ \mathrm{Cl}_{\mathrm{II}}-\mathrm{O} & 3.30 \\ \mathrm{O}-\mathrm{O} & 3.94 \\ \mathrm{O}-\mathrm{O} & 4.27\end{array}$

$\begin{array}{ll}\mathrm{Cs}-\mathrm{O} & 3.78 \\ \mathrm{Cs}-\mathrm{O} & 3.84 \\ \mathrm{Cs}-\mathrm{O} & 4.33\end{array}$


Table 1. Observed and calculated structure factors. For those reflexions too weak to be observed, $F_{\mathrm{o}}$ is listed as half the least observable value.

\begin{tabular}{|c|c|c|c|c|c|c|}
\hline$h \quad k \quad l$ & $F_{\mathrm{o}}$ & $F_{\mathrm{c}}$ & & $k \quad l$ & $F_{\mathrm{o}}$ & $F_{\mathrm{c}}$ \\
\hline $\begin{array}{lll}0 & 0 & 2\end{array}$ & 138 & -147 & 7 & $\begin{array}{ll}0 & 4\end{array}$ & 62 & -53 \\
\hline $\begin{array}{lll}0 & 0 & 4\end{array}$ & 235 & -230 & 7 & $0 \quad 6$ & 26 & \\
\hline $\begin{array}{lll}0 & 0 & 6\end{array}$ & 28 & 36 & 7 & 08 & 71 & 63 \\
\hline $\begin{array}{lll}0 & 0 & 8\end{array}$ & 153 & 153 & 7 & 010 & $<8$ & 5 \\
\hline $\begin{array}{lll}0 & 0 & 10\end{array}$ & 94 & -102 & 7 & 012 & 43 & -40 \\
\hline $\begin{array}{lll}0 & 0 & 12\end{array}$ & $<8$ & 16 & 8 & $\begin{array}{ll}0 & 0\end{array}$ & 103 & 101 \\
\hline $\begin{array}{lll}0 & 0 & 14\end{array}$ & $<8$ & 8 & 8 & $\begin{array}{ll}0 & 2\end{array}$ & 60 & -58 \\
\hline $\begin{array}{lll}0 & 0 & 16\end{array}$ & $<8$ & 1 & 8 & $\begin{array}{ll}0 & 4\end{array}$ & 15 & -16 \\
\hline $\begin{array}{lll}1 & 0 & 2\end{array}$ & 156 & -163 & 8 & $\begin{array}{ll}0 & 6\end{array}$ & 22 & 21 \\
\hline $\begin{array}{lll}1 & 0 & 4\end{array}$ & 125 & 114 & 8 & $\begin{array}{ll}0 & 8\end{array}$ & 34 & \\
\hline $\begin{array}{lll}1 & 0 & 6\end{array}$ & 57 & 75 & 8 & 010 & 57 & -61 \\
\hline $\begin{array}{lll}1 & 0 & 8\end{array}$ & 120 & -115 & 8 & 012 & 15 & \\
\hline 1010 & $<8$ & -9 & 9 & $\begin{array}{ll}0 & 2\end{array}$ & 111 & -99 \\
\hline $\begin{array}{lll}1 & 0 & 12\end{array}$ & 68 & 70 & 9 & $\begin{array}{ll}0 & 4\end{array}$ & 15 & \\
\hline $1 \quad 0 \quad 14$ & $<8$ & -13 & 9 & 06 & 65 & 59 \\
\hline $\begin{array}{lll}1 & 0 & 16\end{array}$ & 22 & -26 & 9 & $\begin{array}{ll}0 & 8\end{array}$ & 15 & -28 \\
\hline $\begin{array}{lll}2 & 0 & 0\end{array}$ & 70 & -81 & 9 & 010 & 15 & -17 \\
\hline $\begin{array}{lll}2 & 0 & 2\end{array}$ & $<8$ & -16 & 10 & $\begin{array}{ll}0 & 0\end{array}$ & $<8$ & -15 \\
\hline $\begin{array}{lll}2 & 0 & 4\end{array}$ & 183 & 184 & 10 & $\begin{array}{ll}0 & 2\end{array}$ & $<8$ & -8 \\
\hline $\begin{array}{lll}2 & 0 & 6\end{array}$ & 174 & -172 & 10 & $\begin{array}{ll}0 & 4\end{array}$ & 51 & 53 \\
\hline $\begin{array}{lll}2 & 0 & 8\end{array}$ & 15 & 10 & 10 & 06 & 63 & -55 \\
\hline $\begin{array}{lll}2 & 0 & 10\end{array}$ & 51 & 55 & 10 & $\begin{array}{ll}0 & 8\end{array}$ & $<8$ & 7 \\
\hline $\begin{array}{lll}2 & 0 & 12\end{array}$ & $<8$ & 20 & 10 & 010 & 22 & 15 \\
\hline $2 \quad 0 \quad 14$ & 62 & -63 & 11 & $\begin{array}{ll}0 & 2\end{array}$ & 31 & 30 \\
\hline $\begin{array}{lll}3 & 0 & 2\end{array}$ & 365 & 321 & 11 & $\begin{array}{ll}0 & 4\end{array}$ & 22 & -20 \\
\hline $\begin{array}{lll}3 & 0 & 4\end{array}$ & 62 & -73 & 11 & $\begin{array}{ll}0 & 6\end{array}$ & $<8$ & -16 \\
\hline $\begin{array}{lll}3 & 0 & 6\end{array}$ & 170 & -161 & 11 & $\begin{array}{ll}0 & 8\end{array}$ & 22 & 24 \\
\hline $\begin{array}{lll}3 & 0 & 8\end{array}$ & 62 & 73 & 12 & $\begin{array}{ll}0 & 0\end{array}$ & 72 & \\
\hline $\begin{array}{lll}3 & 0 & 10\end{array}$ & 40 & 43 & 12 & $\begin{array}{ll}0 & 2\end{array}$ & 22 & -23 \\
\hline $\begin{array}{lll}3 & 0 & 12\end{array}$ & 22 & -41 & 12 & $\begin{array}{ll}0 & 4\end{array}$ & 26 & -22 \\
\hline $\begin{array}{lll}3 & 0 & 14\end{array}$ & $<8$ & 6 & 0 & 10 & 45 & 42 \\
\hline $\begin{array}{lll}4 & 0 & 0\end{array}$ & 202 & 198 & 0 & 12 & 62 & 57 \\
\hline $\begin{array}{lll}4 & 0 & 2\end{array}$ & 131 & -114 & 0 & 14 & 161 & -168 \\
\hline $\begin{array}{lll}4 & 0 & 4\end{array}$ & 26 & -36 & 0 & 16 & 107 & 114 \\
\hline $\begin{array}{lll}4 & 0 & 6\end{array}$ & 60 & 51 & 0 & 18 & $<15$ & -12 \\
\hline $\begin{array}{lll}4 & 0 & 8\end{array}$ & 56 & 68 & 0 & 110 & $<15$ & - 6 \\
\hline $\begin{array}{lll}4 & 0 & 10\end{array}$ & 113 & -110 & 0 & 112 & $<15$ & -25 \\
\hline $\begin{array}{lll}4 & 0 & 12\end{array}$ & 26 & 29 & 0 & 114 & 33 & 37 \\
\hline $\begin{array}{lll}4 & 0 & 14\end{array}$ & 22 & 21 & 0 & 116 & $<15$ & -22 \\
\hline $\begin{array}{lll}5 & 0 & 2\end{array}$ & 105 & -103 & 0 & 20 & 180 & 189 \\
\hline $\begin{array}{lll}5 & 0 & 4\end{array}$ & 76 & 71 & 0 & $\begin{array}{ll}2 & 2\end{array}$ & 153 & -142 \\
\hline $\begin{array}{ccc}5 & 0 & 6\end{array}$ & 46 & 53 & 0 & 24 & $<15$ & -17 \\
\hline $\begin{array}{lll}5 & 0 & 8\end{array}$ & 82 & -81 & 0 & 26 & 90 & 78 \\
\hline $\begin{array}{lll}5 & 0 & 10\end{array}$ & $<8$ & -7 & 0 & 28 & 42 & \\
\hline $\begin{array}{lll}5 & 0 & 12\end{array}$ & 46 & 50 & 0 & 210 & 131 & -132 \\
\hline $\begin{array}{llll}5 & 0 & 14\end{array}$ & $<8$ & -10 & 0 & 212 & 36 & 34 \\
\hline $\begin{array}{lll}6 & 0 & 0\end{array}$ & 158 & -150 & 0 & 214 & 33 & 32 \\
\hline $\begin{array}{lll}6 & 0 & 2\end{array}$ & 22 & -21 & 0 & $\begin{array}{ll}3 & 0\end{array}$ & 61 & 68 \\
\hline $\begin{array}{lll}6 & 0 & 4\end{array}$ & 217 & 194 & 0 & 32 & $<15$ & 13 \\
\hline $\begin{array}{lll}6 & 0 & 6\end{array}$ & 83 & -90 & 0 & 34 & 132 & -136 \\
\hline $\begin{array}{lll}6 & 0 & 8\end{array}$ & 37 & -32 & 0 & 36 & 111 & 117 \\
\hline $\begin{array}{lll}6 & 0 & 10\end{array}$ & $<8$ & 16 & 0 & 38 & $<15$ & -3 \\
\hline $\begin{array}{lll}6 & 0 & 12\end{array}$ & 22 & 27 & 0 & 310 & 30 & -33 \\
\hline $\begin{array}{lll}6 & 0 & 14\end{array}$ & 15 & -34 & 0 & $\begin{array}{ll}312 \\
1\end{array}$ & $<15$ & -19 \\
\hline $\begin{array}{lll}7 & 0 & 2\end{array}$ & 71 & 72 & 0 & 314 & 36 & 43 \\
\hline
\end{tabular}

Acta Chem. Scand. 16 (1962) No. 8 
Table 1 continued.

\begin{tabular}{|c|c|c|c|c|c|c|c|c|}
\hline$h$ & $k l$ & $F_{\mathrm{o}}$ & $F_{\mathrm{c}}$ & $h$ & $k$ & $l$ & $F_{\mathrm{o}}$ & $F_{\mathrm{c}}$ \\
\hline 0 & 40 & 253 & 233 & 8 & 1 & 0 & $<8$ & 17 \\
\hline 0 & 42 & 78 & -65 & 10 & 1 & 0 & 43 & -54 \\
\hline 0 & 44 & 122 & -110 & 12 & 1 & 0 & $<8$ & 1 \\
\hline 0 & 46 & 36 & 25 & 2 & 2 & 0 & 214 & -196 \\
\hline 0 & 48 & 87 & 82 & 4 & 2 & 0 & 279 & 258 \\
\hline 0 & 410 & 60 & -61 & 6 & 2 & 0 & 16 & -24 \\
\hline 0 & 412 & $<15$ & 11 & 8 & 2 & 0 & 135 & 127 \\
\hline 0 & 414 & $<15$ & 7 & 10 & 2 & 0 & 51 & -42 \\
\hline 0 & 50 & 82 & 70 & 12 & 2 & 0 & 34 & 32 \\
\hline 0 & $\begin{array}{ll}5 & 2\end{array}$ & $<15$ & 12 & 2 & 3 & 0 & 151 & -156 \\
\hline 0 & $\begin{array}{ll}5 & 4\end{array}$ & 102 & -93 & 4 & 3 & 0 & 50 & 45 \\
\hline 0 & 56 & 45 & 41 & 6 & 3 & 0 & 84 & -96 \\
\hline 0 & 58 & $<15$ & 13 & 8 & 3 & 0 & $<8$ & 16 \\
\hline 0 & 510 & $<15$ & -3 & 10 & 3 & 0 & 50 & -47 \\
\hline 0 & $\begin{array}{ll}512 \\
\end{array}$ & $<15$ & -12 & 12 & 3 & 0 & 16 & 2 \\
\hline 0 & $\begin{array}{ll}6 & 0\end{array}$ & 108 & 98 & 2 & 4 & 0 & 90 & -93 \\
\hline 0 & $\begin{array}{ll}6 & 2\end{array}$ & 50 & -44 & 4 & 4 & 0 & 132 & 134 \\
\hline 0 & $\begin{array}{ll}6 & 4\end{array}$ & 60 & -52 & 6 & 4 & 0 & 95 & -90 \\
\hline 0 & $\begin{array}{ll}6 & 6\end{array}$ & 62 & 57 & 8 & 4 & 0 & 84 & 75 \\
\hline 0 & $\begin{array}{ll}6 & 8\end{array}$ & 33 & 32 & 10 & 4 & 0 & 22 & -20 \\
\hline 0 & 610 & 71 & -63 & 2 & 5 & 0 & 87 & -85 \\
\hline 0 & 612 & $<15$ & 7 & 4 & 5 & 0 & 16 & 29 \\
\hline 0 & 70 & $<15$ & 12 & 6 & 5 & 0 & 76 & -74 \\
\hline 0 & 72 & $<15$ & -11 & 8 & 5 & 0 & 16 & 15 \\
\hline 0 & $\begin{array}{ll}7 & 4\end{array}$ & $<15$ & -14 & 10 & 5 & 0 & $<8$ & -24 \\
\hline 0 & 76 & 42 & 35 & 2 & 6 & 0 & 84 & -85 \\
\hline 0 & 78 & $<15$ & -2 & 4 & 6 & 0 & 98 & 96 \\
\hline 0 & 710 & $<15$ & -25 & 6 & 6 & 0 & 50 & -44 \\
\hline 0 & 80 & 56 & 59 & 8 & 6 & 0 & 54 & 51 \\
\hline 0 & $\begin{array}{ll}8 & 2\end{array}$ & $<15$ & -9 & 10 & 6 & 0 & 37 & -26 \\
\hline 0 & 84 & 45 & -49 & 2 & 7 & 0 & 54 & -58 \\
\hline 0 & 86 & $<15$ & 26 & 4 & 7 & 0 & 43 & 44 \\
\hline 0 & 88 & $<15$ & 18 & 6 & 7 & 0 & $<8$ & -12 \\
\hline 0 & $\begin{array}{ll}9 & 0\end{array}$ & 45 & 48 & 8 & 7 & 0 & 22 & 23 \\
\hline 0 & $\begin{array}{ll}9 & 2\end{array}$ & $<15$ & -9 & 2 & 8 & 0 & 57 & -53 \\
\hline 0 & $\begin{array}{ll}9 & 4 \\
9\end{array}$ & $<15$ & -28 & 4 & 8 & 0 & 43 & 41 \\
\hline 0 & $\begin{array}{ll}9 & 6\end{array}$ & $<15$ & 3 & 6 & 8 & 0 & 37 & -41 \\
\hline 01 & 100 & 22 & 18 & 8 & 8 & 0 & 16 & 24 \\
\hline $\begin{array}{llll}0 & 1 \\
0 & 0\end{array}$ & $\begin{array}{ll}10 & 2\end{array}$ & $<15$ & 0 & 2 & 9 & 0 & $<8$ & - 7 \\
\hline 2 & 10 & 236 & -226 & 4 & 9 & 0 & $<8$ & 15 \\
\hline 4 & 10 & $\begin{array}{r}26 \\
193\end{array}$ & 48 & 6 & 9 & 0 & 22 & $\begin{array}{l}-23 \\
-20\end{array}$ \\
\hline 6 & 10 & 123 & -121 & 2 & 10 & 0 & 26 & -36 \\
\hline
\end{tabular}

\section{DISCUSSION}

The manganese atom lies near the centre of a distorted octahedron having four chlorine atoms and two oxygen atoms at the corners. Two chlorine atoms are placed on opposite sides of a trapezium consisting of two oxygen atoms and two chlorine atoms. These six atoms form a cis-octahedral coordination around the manganese atom. The octahedrons sharing corners are arrayed in zig-zag chains along the (100)-direction (Fig. 3). The octahedrons are connected through the cesium-atoms in such a way that each Cs-atom is surrounded by eight

Acta Chem. Scand. 16 (1962) No. 8 


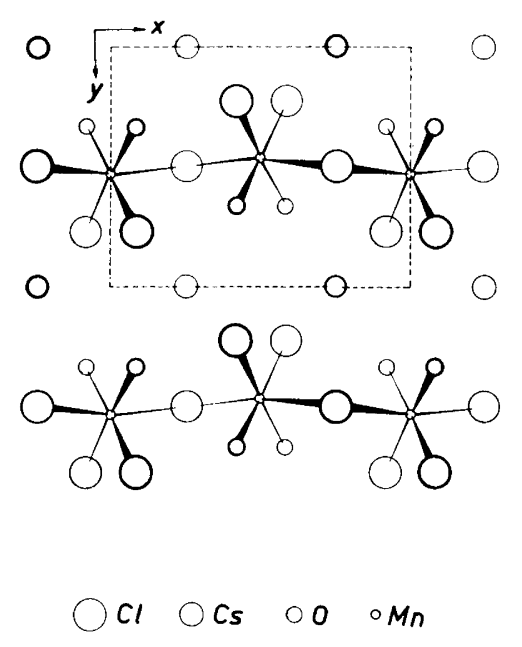

Fig. 3. Projection of the unit cell on (001).
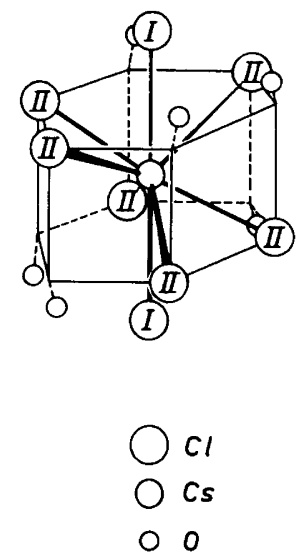

Fig. 4. The coordination around the cesium-atom.

chlorine atoms as nearest neighbours. Next-nearest neighbours are six oxygen atoms. These fourteen atoms form a coordination reminding of a distorted body-centered cubic packing (Fig. 4). The interatomic distances between Mn and $\mathrm{Cl}$ and between $\mathrm{Mn}$ and $\mathrm{O}$ are somewhat smaller than the corresponding distances in $\mathrm{MnCl}_{2}, 2 \mathrm{H}_{2} \mathrm{O}$ found by Vajnstejn ${ }^{7}$. In $\mathrm{MnCl}_{2}, 2 \mathrm{H}_{2} \mathrm{O}, \mathrm{Mn}-\mathrm{Cl}$ is $2.57 \AA$ and $2.60 \AA$ and $\mathrm{Mn}-\mathrm{O}$ is $2.19 \AA$ and the coordination around $\mathrm{Mn}$ is trans-octahedral. In $\mathrm{K}_{2} \mathrm{Mn}\left(\mathrm{SO}_{4}\right)_{2}, 4 \mathrm{H}_{2} \mathrm{O}$ analysed by Schneider ${ }^{8} \mathrm{Mn}$ is surrounded by six oxygen atoms in a distance varying from $2.08 \AA$ to $2.17 \AA$.

Acknowledgement. The Danish State Science Foundation has covered the cost of the Hägg-Laurent computer and the programming and the computations on DASK. We express our thanks to the director of Regnecentralen, N. I. Bech for his active and willing cooperation in the solution of our computational problems, and to Mr. Finn Larsen for carrying out the electronic computations.

\section{REFERENCES}

1. Andersen, P. 9. Nordiske Kemikermøde, Århus 1956, p. 7.

2. Vermande, J. Pharmaceutisch Weekblad 33 (1918) 1131.

3. Nelson, J. B., Riley, D. P. Proc. Phys. Soc. 57 (1945) 160.

4. Frank, V. J. Sci. Instr. 34 (1957) 210.

5. Westman, S., Blomqvist, G. and Åsbrink, S. Arkiv Kemi 14 (1959) 535.

6. Forsyth, J. B. and Wells, M. Acta Cryst. 12 (1959) 412.

7. Vajnstejn, B. K. Doklady Akad. Nauk. SSSR 83 (1952) 227.

8. Schneider. W. Acta Cryst. 14 (1961) 784.

Received April 25, 1962. 